\title{
Relationship between Macro and Micronutrients Profile with Fungal Flora of Rhizosphere Soils from Wheat, Maize and Sorghum Fields of Baramati Area
}

\author{
Rohit Taware, Pooja Kadam, Anuja Shende, Anuradha Bhosale, \\ M. B. Kanade* and S. J. Chavan
}

Department of Botany, Tuljaram Chaturchand College of Arts, Science and Commerce, Baramati (Autonomous), Dist. Pune, Maharashtra, India

*Corresponding author

\section{A B S T R A C T}

The present study emphasizes on profile of macro and micronutrients and fungal flora of wheat, maize and Sorghum rhizosphere soils at sowing, flowering and harvesting stages of crops from Baramati area of Pune district of Maharashtra. From the studied fields total 14 fungal genera and 17 species belonging to fungal group Mastigomycotina (03),

Keywords Zygomycotina (02), Ascomycotina (01), Basidiomycotina (01) and Deuteromycotina (07)

Wheat, Maize, Sorghum, Soil macro and Micronutrients and soil fungal flora

Article Info

Accepted: 12 November 2020 Available Online: 10 December 2020 were reported. The wheat field soils of Sangavi, Pandare and Tawadi area showed deficiency of nitrogen and phosphorus, very high amount of potassium and calcium, sufficient amount of magnesium, sulphur at lowest amount, the micronutrients iron, manganese, zinc and copper reported at stress amount compared to standard values. The fungi isolated from wheat fields were majority saprophytic and Alternaria and Fusarium reported as pathogenic. Macro and micronutrients profile of maize was studied from Deulgaon Rasal, Sangavi and Nimbodi fields. Like wheat fields, maize field soils also showed deficiency values of nitrogen, phosphorous and sulphur. The amount of potassium, calcium and magnesium was very high, the concentration of iron, manganese, zinc and copper were extremely less compared with standard values. The parasitic fungi Phytophthora, Helminthosporium, Fusarium and Alternaria were most frequently reported from all studied maize fields. The Sorghum field soils studied from Malad, Deulgaon Rasal and Jalgaon reported the deficiency level of nitrogen and phosphorous, the quantity of potassium was at very high level, the amount of calcium and magnesium was adequate and the concentration of sulphur, iron, manganese, zinc and copper were at declined level. Almost saprophytic fungi Penicillium, Aspergillus and Mucor and pathogenic fungi Helminthosporium and Fusarium were reported frequently from rhizosphere soils of Sorghum.

\section{Introduction}

A healthy soil is an ecosystem teeming with various microbes that perform various vital functions including deterioration and decomposition of organic matter (Doran, 2002) which helps in soil nutrient enrichment. The fungal flora is one of the important and 
most successful inhabitants of soil (Sun et al., 2005) they have ability to product a wide variety of extracellular enzymes and convert dead and organic matter into biomass, carbon dioxide and other macro and micronutrients. The fungal populations are strongly influenced by diversity of the plants community and in return affect plant growth through mutualism, pathogenicity and their affect on nutrient availability and cycling (Wagg et al., 2014). Some pathogenic fungi can disturb the nutrients cycle of soil (Frac et al., 2018), while few can cause economic losses of crops. From the available literature it is confirmed that there should be interrelationship between macro and micronutrients profile available in the soil with the fungal flora inhabitants in the soil. In this connection, the present investigation particularly focusing on the relationship between fungal flora available in the soil with soil nutrients profile of wheat, maize and Sorghum fields.

\section{Materials and Methods}

The prime objective of present work is to study the interaction of soil fungal flora and macro and micronutrients profile of the soil with respect to common cereal crops viz. wheat, maize and Sorghum cultivated in Baramati area of Pune district of Maharashtra. In this connection we have collected soil samples from different localities at different stages of the crop plants like sowing, flowering and harvesting stages. The soil samples were collected during the October, 2019 to February, 2020. Particularly rhizosphere soil samples were collected in sterile polythene bags from respective crop fields and brought to laboratory. The soils were clean to remove stones, gravels and other coarse residues, large pieces of soil were broken by hand. The air dried soil samples were crushed in mortal and pestle and sieved through $2 \mathrm{~mm}$ sieve. These soil samples were used further for study of soil fungal flora and macro and micronutrient profile of soil. The macro and micronutrients were analysed from Krishi Vidnyan Kendra, Baramati (KVK). Soil fungi were isolated by using serial dilution method (Aneja, 2003). The Czapek's Dox Agar [CDA] (Kanade et al., 2018) and Potato Dextrose Agar [PDA] (Shitole et al., 2019) media supplemented by Penicillin antibiotic.

Inoculated plates were kept for incubation at room temperature for 7 days. At the time of incubation fungal growth was observed regularly and observations were noticed. After 7days of incubation the photographs of plates were taken. Isolated fungal colonies were used for preparation of slides. Slides were prepared using cotton blue stain and lactophenol as mounting medium. Slides were observed under light microscope and microphotography was also done. Fungi were identified on the basis of morphological characters of spores by using standard literature (Nagamani et al., 2006).

\section{Results and Discussion}

The present study was carried out to study the profile of inorganic constituents and soil mycoflora of wheat, maize and Sorghum rhizosphere soils from Baramati area of Pune district of Maharashtra. In connection with this, total 14 fungal genera and 17 species belonging to fungal group Mastigomycotina (03), Zygomycotina (02), Ascomycotina (01), Basidiomycotina (01) and Deuteromycotina (07) were recorded (Table 1). The percentage contributions of isolated fungal members were noticed as Mucor, Aspergillus and Fusarium (100\%), Rhizopus (88.88\%), Phytophthora, Alternaria and Penicillium (55.55\%), Helminthosporium (44.44\%), Cryptococcus (33.33\%), Pythium and Trichoderma (22.22\%) and Achlya, Cladosporium and Rhizoctonia (11.11\%). It is 
investigating to note that, the fungi Mucor, Aspergillus and Fusarium were reported from rhizosphere soils of all crops plants concerning with all the stages of crop plants viz. sowing, flowering and harvesting, on the contrary fungus Achlya was reported from the maize fields and Cladosporium and Rhizoctonia were reported from wheat crop soil samples only (Table 1).

The macro and micronutrients amount was assessed from the rhizosphere soil of wheat, maize and Sorghum crop fields at sowing, flowering and harvesting stages of the respective crops depicted in Table 2, 3 and 4 . In case of macronutrients, nitrogen $(\mathrm{N})$, phosphorus $(\mathrm{P})$, potassium $(\mathrm{K})$, calcium $(\mathrm{Ca})$, magnesium $(\mathrm{Mg})$ and sulphur $(\mathrm{S})$ were studied. On the other hand micronutrients iron $(\mathrm{Fe})$, manganese $(\mathrm{Mn})$, zinc $(\mathrm{Zn})$ and copper $(\mathrm{Cu})$ were analyzed.

In wheat field soils of Sangavi, Pandare and Tawadi area showed deficiency of nitrogen and phosphorus (Table 2). In Baramati area the level of sufficient amount of nitrogen is $561-700 \mathrm{~kg} / \mathrm{ha}$, but from collected soil samples maximum amount of nitrogen was reported as
$112 \mathrm{~kg} / \mathrm{ha}$, which was very low compared to standard values. The amount of potassium was very high in all collected soil samples compared to standard profile $(251-300 \mathrm{~kg} / \mathrm{ha})$ and more amount of potassium was noted from Sangavi soil i.e. $1589 \mathrm{~kg} / \mathrm{ha}$ at sowing stage of wheat. In case of calcium it was also very high in amount like potassium. The adequate amount of calcium in our area is $11+\mathrm{kg} / \mathrm{ha}$, but we recorded $54.9 \mathrm{~kg} / \mathrm{ha}$ calcium concentration from Pandare soil at flowering stage of wheat. Magnesium content was reported as in sufficient amount i.e. more than $70 \mathrm{ppm}$ from all the crop plants fields. On the contrary sulphur was reported as less than lowest amount i.e. 6-10ppm from all studied crop fields (Table 2). The profile of micronutrients $v i z$. iron $(\mathrm{Fe})$, manganese $(\mathrm{Mn})$, zinc $(\mathrm{Zn})$ and copper $(\mathrm{Cu})$ was very low compared to standard values (i.e. more than 10ppm, 05ppm, 1ppm and 0.4ppm respectively). Maximum amount of iron (0.73ppm), manganese (0.29ppm), Zinc $(0.11 \mathrm{ppm})$ and copper $(0.15 \mathrm{ppm})$ were recorded from Pandare fields at sowing stage of wheat, but these values are very less compared to standard values (Table 2).

Table.1 List of fungi isolated from wheat, maize and Sorghum fields using CDA and PDA media

\begin{tabular}{|c|c|c|c|c|c|c|c|c|c|c|}
\hline \multirow[t]{2}{*}{ Sr.No. } & \multirow[t]{2}{*}{ Name of fungi } & \multicolumn{3}{|c|}{ Wheat fields } & \multicolumn{3}{|c|}{ Maize fields } & \multicolumn{3}{|c|}{ Sorghum fields } \\
\hline & & $\mathbf{S}$ & $\mathbf{P}$ & $\mathbf{T}$ & D & $\mathbf{S}$ & $\mathbf{N}$ & $\mathbf{M}$ & D & $\mathbf{J}$ \\
\hline 1. & Pythium sp. & - & + & + & - & - & - & - & - & - \\
\hline 2. & Phytophthora sp. & - & - & - & + & - & + & + & + & + \\
\hline 3. & Achlya sp. & - & - & - & - & + & - & - & - & - \\
\hline 4. & Rhizopus sp. & + & + & + & + & + & + & + & - & + \\
\hline 5. & Mucor sp. & + & + & + & + & + & + & + & + & + \\
\hline 6. & Trichoderma sp. & + & - & - & - & - & + & - & - & - \\
\hline 7. & Cryptococcus sp. & - & - & + & - & - & - & + & + & - \\
\hline 8. & Cladosporium sp. & - & - & + & - & - & - & - & - & - \\
\hline 9. & Alternaria sp. & - & + & - & + & + & - & + & + & - \\
\hline 10. & Aspergillus sp. & + & + & + & + & + & + & + & + & + \\
\hline 11. & Fusarium sp. & + & + & + & + & + & + & + & + & + \\
\hline 12. & Penicillium sp. & + & + & + & + & - & - & + & - & - \\
\hline 13. & Helminthosporium sp. & - & - & + & + & + & - & + & + & - \\
\hline 14. & Rhizoctonia sp. & - & - & + & - & - & - & - & - & - \\
\hline
\end{tabular}

(S : Sangavi, P : Pandare, T : Tawadi, D : Deulgaon Rasal, N : Nimbodi, M : Malad, J : Jalgaon crop fields, + present, - absent) 
Table.2 Profile of macro and micronutrients and isolated fungi from wheat field soils

\begin{tabular}{|c|c|c|c|c|c|c|c|c|c|c|c|c|}
\hline \multirow[t]{2}{*}{ Area } & \multirow{2}{*}{$\begin{array}{c}\text { Soil samples } \\
\text { at }\end{array}$} & \multicolumn{6}{|c|}{ Macronutrients } & \multicolumn{4}{|c|}{ Micronutrients } & \multirow[t]{2}{*}{ Fungi } \\
\hline & & $\mathbf{N}^{*}$ & $\mathbf{P}^{*}$ & $\mathbf{K}^{*}$ & $\mathrm{Ca}^{*}$ & Mg\# & S\# & Fe\# & Mn\# & Zn\# & $\mathrm{Cu} \#$ & \\
\hline \multirow[t]{3}{*}{ Sangavi } & $\begin{array}{l}\text { Sowing } \\
\text { stage }\end{array}$ & 37.63 & 3.2 & 1589 & 32 & 97 & 2.9 & 0.34 & 0.15 & 0.06 & 0.07 & $\begin{array}{l}\text { Aspergillus sp. } \\
\text { Mucor } \mathrm{sp} .\end{array}$ \\
\hline & $\begin{array}{l}\text { Flowering } \\
\text { stage }\end{array}$ & 100 & 3.6 & 588 & 32.5 & 48 & 5.86 & 0.43 & 0.18 & 0.06 & 0.08 & $\begin{array}{l}\text { Trichoderma } \mathrm{sp} . \\
\text { Fusarium } \mathrm{sp} . \\
\text { Penicillium } \mathrm{sp} . \\
\text { Aspergillus } \mathrm{sp} .\end{array}$ \\
\hline & $\begin{array}{l}\text { Harvesting } \\
\text { stage }\end{array}$ & 87.88 & 2.18 & 485 & 43.5 & 80.5 & 7.81 & 0.36 & 0.15 & 0.06 & 0.08 & $\begin{array}{l}\text { Aspergillus sp. } \\
\text { Rhizopus sp. }\end{array}$ \\
\hline \multirow[t]{3}{*}{ Pandare } & $\begin{array}{l}\text { Sowing } \\
\text { stage }\end{array}$ & 75.26 & 5.08 & 980 & 47.5 & 82 & 5.15 & 0.73 & 0.29 & 0.11 & 0.15 & $\begin{array}{l}\text { Rhizopus sp. } \\
\text { Mucor sp. } \\
\text { Alternaria sp. } \\
\text { Penicillium sp. } \\
\text { Aspergillus sp. }\end{array}$ \\
\hline & $\begin{array}{l}\text { Flowering } \\
\text { stage }\end{array}$ & 50.17 & 6.19 & 833 & 54.9 & 23.5 & 5.1 & 0.52 & 0.22 & 0.08 & 0.09 & $\begin{array}{l}\text { Fusarium sp. } \\
\text { Alternaria sp. } \\
\text { Pythium sp. } \\
\text { Rhizopus sp. }\end{array}$ \\
\hline & $\begin{array}{l}\text { Harvesting } \\
\text { stage }\end{array}$ & 25.08 & 4.46 & 709 & 39 & 20.5 & 3.82 & 0.5 & 0.23 & 0.06 & 0.1 & $\begin{array}{l}\text { Aspergillus sp. } \\
\text { Rhizopus sp. }\end{array}$ \\
\hline \multirow[t]{3}{*}{ Tawadi } & $\begin{array}{l}\text { Sowing } \\
\text { stage }\end{array}$ & 112 & 3.04 & 1197 & 31.5 & 64.5 & 4.83 & 0.51 & 0.15 & 0.05 & 0.14 & $\begin{array}{l}\text { Aspergillus sp. } \\
\text { Rhizopus sp. } \\
\text { Fusarium sp. }\end{array}$ \\
\hline & $\begin{array}{l}\text { Flowering } \\
\text { stage }\end{array}$ & 100 & 2.85 & 581 & 32.5 & 70.5 & 2.58 & 0.42 & 0.16 & 0.06 & 0.11 & $\begin{array}{l}\text { Aspergillus sp. } \\
\text { Mucor } \mathrm{sp} . \\
\text { Penicillium sp. } \\
\text { Cladosporium sp. } \\
\text { Cryptococcus sp. }\end{array}$ \\
\hline & $\begin{array}{l}\text { Harvesting } \\
\text { stage }\end{array}$ & 87.88 & 1.23 & 645 & 39. & 91 & 5.77 & 0.44 & 0.27 & 0.06 & 0.13 & $\begin{array}{l}\text { Rhizoctonia sp. } \\
\text { Aspergillus sp. } \\
\text { Helminthosporium } \\
\text { sp. } \\
\text { Pythium sp. }\end{array}$ \\
\hline
\end{tabular}


Table.3 Profile of macro and micronutrients and isolated fungi from maize field soils

\begin{tabular}{|c|c|c|c|c|c|c|c|c|c|c|c|c|}
\hline \multirow[t]{2}{*}{ Area } & \multirow{2}{*}{$\begin{array}{c}\text { Soil } \\
\text { samples }\end{array}$} & \multicolumn{6}{|c|}{ Macronutrients } & \multicolumn{4}{|c|}{ Micronutrients } & \multirow[t]{2}{*}{ Fungi } \\
\hline & & $\mathbf{N}^{*}$ & P* & $\mathbf{K}^{*}$ & Ca* & Mg\# & S\# & Fe\# & Mn\# & Zn\# & Cu\# & \\
\hline \multirow[t]{3}{*}{$\begin{array}{c}\text { Deulgaon } \\
\text { Rasal }\end{array}$} & $\begin{array}{l}\text { Sowing } \\
\text { stage }\end{array}$ & 87.88 & 4.69 & 448 & 34.5 & 47.5 & 0.38 & 0.53 & 0.38 & 0.05 & 0.26 & $\begin{array}{l}\text { Aspergillus sp. } \\
\text { Mucor } \mathrm{sp} . \\
\text { Phytophthora sp. } \\
\text { Rhizopus } \mathrm{sp} .\end{array}$ \\
\hline & $\begin{array}{l}\text { Flowering } \\
\text { stage }\end{array}$ & 37.68 & 3.6 & 4655 & 51.5 & 75 & 0.12 & 0.59 & 0.12 & 0.03 & 0.14 & $\begin{array}{l}\text { Helminthosporium } \\
\text { sp. } \\
\text { Fusarium sp. } \\
\text { Penicillium sp. } \\
\text { Aspergillus sp. }\end{array}$ \\
\hline & $\begin{array}{l}\text { Harvesting } \\
\text { stage }\end{array}$ & 122 & 3.44 & 510 & 34 & 49.5 & 0.27 & 0.47 & 0.27 & 0.05 & 0.23 & $\begin{array}{l}\text { Phytophthora sp. } \\
\text { Alternaria sp. } \\
\text { Fusarium sp. }\end{array}$ \\
\hline \multirow[t]{3}{*}{ Sangavi } & $\begin{array}{l}\text { Sowing } \\
\text { stage }\end{array}$ & 12.54 & 5.64 & 534 & 40.5 & 49.5 & 0.45 & 0.74 & 0.45 & 0.07 & 0.23 & $\begin{array}{l}\text { Alternaria sp. } \\
\text { Achlya } \mathrm{sp} . \\
\text { Aspergillus sp. }\end{array}$ \\
\hline & $\begin{array}{l}\text { Flowering } \\
\text { stage }\end{array}$ & 50.17 & 5.72 & 561 & 57 & 95 & 0.5 & 0.49 & 0.5 & 0.06 & 0.31 & $\begin{array}{l}\text { Helminthosporium } \\
\text { sp. } \\
\text { Fusarium sp. } \\
\text { Mucor sp. } \\
\text { Aspergillus sp. }\end{array}$ \\
\hline & $\begin{array}{l}\text { Harvesting } \\
\text { stage }\end{array}$ & 25.08 & 4.93 & 523 & 57.5 & 77.5 & 0.17 & 0.48 & 0.17 & 0.01 & 0.1 & $\begin{array}{l}\text { Alternaria sp. } \\
\text { Rhizopus sp. } \\
\text { Mucor sp. }\end{array}$ \\
\hline \multirow[t]{3}{*}{ Nimbodi } & $\begin{array}{l}\text { Sowing } \\
\text { stage }\end{array}$ & 12.54 & 3.75 & 323 & 39.5 & 46.5 & 0.46 & 0.49 & 0.46 & 0.21 & 0.21 & $\begin{array}{l}\text { Rhizopus sp. } \\
\text { Fusarium sp. } \\
\text { Trichoderma sp. }\end{array}$ \\
\hline & $\begin{array}{l}\text { Flowering } \\
\text { stage }\end{array}$ & 87.88 & 2.33 & 377 & 31.5 & 46.5 & 0.1 & 0.61 & 0.1 & 0.03 & 0.13 & $\begin{array}{l}\text { Aspergillus sp. } \\
\text { Mucor sp. } \\
\text { Trichoderma sp. }\end{array}$ \\
\hline & $\begin{array}{l}\text { Harvesting } \\
\text { stage }\end{array}$ & 112 & 2.96 & 382 & 31 & 50 & 0.22 & 0.32 & 0.22 & 0.03 & 0.03 . & $\begin{array}{l}\text { Mucor sp. } \\
\text { Phytophthora sp. } \\
\text { Aspergillus sp. }\end{array}$ \\
\hline
\end{tabular}

*kilogram/hectare, \# parts/million 
Table.4 Profile of macro and micronutrients and isolated fungi from Sorghum field soils

\begin{tabular}{|c|c|c|c|c|c|c|c|c|c|c|c|c|}
\hline \multirow[t]{2}{*}{ Area } & \multirow{2}{*}{$\begin{array}{c}\text { Soil } \\
\text { samples } \\
\text { at }\end{array}$} & \multicolumn{6}{|c|}{ Macronutrients } & \multicolumn{4}{|c|}{ Micronutrients } & \multirow[t]{2}{*}{ Fungi } \\
\hline & & $\mathbf{N}^{*}$ & $\mathbf{P} *$ & $\mathbf{K}^{*}$ & $\mathbf{C a}^{*}$ & Mg\# & S\# & Fe\# & Mn\# & Zn\# & Cu\# & \\
\hline \multirow[t]{3}{*}{ Malad } & $\begin{array}{l}\text { Sowing } \\
\text { stage }\end{array}$ & 37.63 & 2.65 & 10.26 & 26 & 63.5 & 2.58 & 0.43 & 0.05 & 0.06 & 0.04 & $\begin{array}{l}\text { Aspergillus sp. } \\
\text { Mucor sp. } \\
\text { Rhizopus } \mathrm{sp} .\end{array}$ \\
\hline & $\begin{array}{l}\text { Flowering } \\
\text { stage }\end{array}$ & 100 & 2.25 & 1360 & 39.5 & 51.5 & 3.18 & 0.55 & 0.15 & 0.06 & 0.07 & $\begin{array}{l}\text { Helminthosporium } \mathrm{sp} . \\
\text { Penicillium } \mathrm{sp} . \\
\text { Fusarium } \mathrm{sp} . \\
\text { Aspergillus } \mathrm{sp} .\end{array}$ \\
\hline & $\begin{array}{l}\text { Harvesting } \\
\text { stage }\end{array}$ & 87.88 & 0.52 & 12.88 & 36.5 & 63 & 4.12 & 0.7 & 0.27 & 0.07 & 0.1 & $\begin{array}{l}\text { Fusarium sp. } \\
\text { Trichoderma sp. } \\
\text { Aspergillus sp. }\end{array}$ \\
\hline \multirow[t]{3}{*}{$\begin{array}{c}\text { Deulgaon } \\
\text { Rasal }\end{array}$} & $\begin{array}{l}\text { Sowing } \\
\text { stage }\end{array}$ & 37.63 & 1.39 & 593 & 48 & 51.5 & 5.86 & 0.3 & 0.9 & 0.05 & 0.06 & $\begin{array}{l}\text { Trichoderma } \mathrm{sp} . \\
\text { Rhizopus } \mathrm{sp} . \\
\text { Mucor } \mathrm{sp} . \\
\text { Aspergillus } \mathrm{sp} .\end{array}$ \\
\hline & $\begin{array}{l}\text { Flowering } \\
\text { stage }\end{array}$ & 12.54 & 1.23 & 542 & 50.5 & 60 & 3.18 & 1.7 & 0.1 & 0.06 & 0.03 & $\begin{array}{l}\text { Helminthosporium sp. } \\
\text { Mucor } \mathrm{sp} . \\
\text { Fusarium } \mathrm{sp} . \\
\text { Trichoderma } \text { sp. }\end{array}$ \\
\hline & $\begin{array}{l}\text { Harvesting } \\
\text { stage }\end{array}$ & 112 & 0.84 & 650 & 45 & 30 & 2.6 & 0.32 & 0.68 & 0.04 & 0.05 & $\begin{array}{l}\text { Fusarium sp. } \\
\text { Aspergillus sp. } \\
\text { Mucor sp. }\end{array}$ \\
\hline \multirow[t]{3}{*}{ Jalgaon } & $\begin{array}{l}\text { Sowing } \\
\text { stage }\end{array}$ & 87.88 & 3.36 & 534 & 63.5 & 132.5 & 1.82 & 0.39 & 0.27 & 0.06 & 0.08 & $\begin{array}{l}\text { Rhizopus sp. } \\
\text { Mucor sp. } \\
\text { Penicillium sp. } \\
\text { Fusarium sp. }\end{array}$ \\
\hline & $\begin{array}{l}\text { Flowering } \\
\text { stage }\end{array}$ & 75.26 & 1 & 18.41 & 31 & 80 & 3.18 & 0.44 & 0.89 & 0.06 & 0.08 & $\begin{array}{l}\text { Aspergillus sp. } \\
\text { Mucor } \mathrm{sp} .\end{array}$ \\
\hline & $\begin{array}{l}\text { Harvesting } \\
\text { stage }\end{array}$ & 50.17 & 8.31 & 19.70 & 39.5 & 42.5 & 4.42 & 0.5 & 1.89 & 0.19 & 0.17 & $\begin{array}{l}\text { Mucor } \mathrm{sp} . \\
\text { Penicillium } \mathrm{sp} . \\
\text { Aspergillus } \mathrm{sp} . \\
\text { Trichoderma } \mathrm{sp} .\end{array}$ \\
\hline
\end{tabular}

*kilogram/hectare, \# parts/million

The fungi isolated from wheat fields were majority saprophytic, on the hands Alternaria and Fusarium reported as pathogenic from Pandare and Tawadi fields at flowering and sowing stages of wheat respectively.

Macro and micronutrient profile of maize was studied from Deulgaon Rasal, Sangavi and Nimbodi fields. The macro and micronutrients profile of studied maize fields was majority near about same. Like wheat fields, maize fields also showed deficiency values of nitrogen, phosphorous and sulphur.
The amount of potassium (4655kg/ha), calcium $(57.5 \mathrm{~kg} / \mathrm{ha})$ and magnesium $(95 \mathrm{ppm})$ was very high compared to standard concentrations (i.e. $251-300 \mathrm{~kg} / \mathrm{ha},>11 \mathrm{~kg} / \mathrm{ha}$ and $>70 \mathrm{ppm}$ respectively). In case of micronutrients, the values of iron $(0.74 \mathrm{ppm})$, manganese $(0.46 \mathrm{ppm})$, zinc $(0.21 \mathrm{ppm})$ and copper (0.31) were remarkably less compared with standard profile (i.e. $>10 \mathrm{ppm},>5 \mathrm{pm}$, $>1 \mathrm{ppm}$ and $>0.4 \mathrm{ppm}$ respectively) (Table 3 ).

The fungi reported from maize fields were majority parasitic. Phytophthora, 
Helminthosporium, Fusarium and Alternaria species were most frequently occurred from all studied maize fields at all crop stages i.e. sowing, flowering and harvesting (Table 3).

In case of Sorghum fields the study areas were Malad, Deulgaon Rasal and Jalgaon. The values of nitrogen and phosphorous showed very less in quantity i.e. near about deficiency level from all the studied areas. Potassium content also showed drastic changes in its quantity. The amount of potassium was sufficient from Deulgaon Rasal Sorghum fields at sowing, flowering and harvesting stages, on the contrary from Malad fields it was high at flowering stages and from Jalgaon fields high at sowing stages. The concentration of calcium and magnesium was adequate amount from all the studied areas at all Sorghum stages (Table 4). The concentration of sulphur, iron, manganese, zinc and copper were again declined at Sorghum fields of all studied areas at all stages of crop plants (sowing, flowering and harvesting).

The isolated fungi from the Sorghum fields were almost saprophytic like Penicillium, Aspergillus, Mucor etc. and pathogenic fungi Helminthosporium and Fusarium were reported frequently from all fields of Sorghum (Table 4).

A soil is the key foundation of crops in agricultural fields where the fungal flora plays vital role in its nutrient profile. In droughts the fungi protects plant root by protective sheath to supply both water and phosphorous (Magdoff and Harold, 2009). According to Muhammad et al., (2016) microbes, bacteria and fungi naturally occurring in soil or supplied as bio-fertilizers, could represent a promising approach to increase nutrients bioavailability and improve soil structure. Anil Kumar et al., (2016) stated that, availability of micronutrients to plants is regulated by various soil factors including such as texture, soil reaction, organic matter, clay content, soil moisture, nutrient interactions in soil, microbial activity, redox potential and aeration. Moreover, they noticed that, exploitation of soil microbes such as micronutrient solubilisers and AM fungi has proven as boon in micronutrient uptake and improving soil quality. Naheeda et al., (2019) concluded that, arbuscular mycorrhizal fungi (AMF) being natural root symbionts, provide essential plant inorganic nutrients to host plants, thereby improving growth and yield under unstressed and stressed regimes. Furthermore they described that, improved resistance to a variety of stresses including drought, salinity, herbivory, temperature, metals, and diseases due to fungal symbiosis.

It is vividly clear from the present results that, the concentration of nitrogen and phosphorous is very low or at deficiency level from all the crop plants at their sowing, flowering and harvesting stages. Level of potassium, calcium and magnesium is sufficient in amount from soils of all studied crop plants. On the contrary profile of sulphur, iron, manganese, zinc and copper showed tremendous inhibitory trend or at deficiency level from wheat, maize and Sorghum fields at all the crop plant stages studied. The macro and microelements of soil plays an important role in healthy and better growth of the crops (Agrios, 2005 and Datnoff et al., 2007), but farmers should not bother about this. Hence before sowing the crop seeds, agriculture soil should be tested from the soil testing laboratories (Bhatt and Sharma, 2011).

The role of soil fungi in healthy growth of crop plants is proven by many workers (Bagyaraj and Ashwin, 2017). They play an important role in deteriorating and decomposition of soil organic matter, maintain soil humans conditions and 
biocontrolling of plant pathogenic fungi (Zifcakova et al., 2016; Treseder and Lennon, 2015). There is strong relationship between amount of macro and micronutrients with population and multiplication of soil mycoflora (Richard et al., 2017). Many saprophytic fungi directly or indirectly are helpful in healthy crop growth (Arriagada et al., 2009a,b). So, farmers should take care of the health of their agriculture soils, avoid unknowingly and indiscriminate use of chemical fertilizers.

\section{References}

Agrios, N.G. (2005). Plant Pathology, $5^{\text {th }}$ ed., Elsevier-Academic Press.

Aneja K. R. (2003). Experiments in Microbiology. Plant Pathology and Biotechnology, New Age International Publication.

Anil Kumar, Choudhary, A. K., Pooniya, V., Suri, V. K., and Singh, U. (2016). Soil factors associated with micronutrient acquisition in crops - biofortification perspective. Book - Biofortification of Food Crops, 159-176.

Arriagada, C., Aranda, E., Sampedro, I., Garcia- Romera, I. and Ocampo, J. A. (2009a). Contribution of the saprobic fungi Trametes versicolor and Trichoderma harzianum and the arbuscular mycorrhizal fungi Glomus deserticola and $G$. claroideum to arsenic tolerance of Eucalyptus globulus. Bioresource Technology, 100(24): 6250-6257.

Arriagada, C., Sampedro, I., Garcia-Romera, I. and Ocampo, J. A. (2009b). Improvement of growth of Eucalyptus globulus and soil biological parameters by amendment with sewage sludge and inoculation with arbuscular mycorrhizal and saprobe fungi. Science of the Total Environmental, 407(17): 4799-4806.
Bagyaraj, D. J. and Ashwin, R. (2017). Soil biodiversity: role in sustainable horticulture. Biodivers. Hortic. Crops, 5: $1-18$.

Bhatt, R. and Sharma, M. (2011). Potassium scenario- a case study in the Kapurthala district of Punjab, India. J Res Punjab Agric Univ, 48: 24-27.

Datnoff, L. E., Elmer, W. H. and Huber, D. M. (2007). Mineral Nutrition and Plant Disease. The American Phytopathological Society, St. Paul, Minnesota, U.S.A.

Doran, J. W. (2002). Soil health of global sustainability: translocating science into practice. Agriculture, Ecosystems and Environment, 88(2): 119-127.

Frac, M., Hannula, S. E., Bełka, B. and Jedryczka, M. (2018). Fungal biodiversity and their role in soil health. Frontiers in Microbiology, 9:707.

Kanade, M. B. Awatade Atul, Gulave Akshay, Wagh Sujit, Kalkute Akshay, Chandankar Sourabh and Waghmare Anjali (2018). Aeromycoflora over jowar and pomegranate fields at Baramati, Dist. Pune (M.S.), Bioscience Discovery, 9(1), 93-96.

Magdoff, F. and Harold, V. ES. (2009). Building soils for better crops. Published by Sustainable Agriculture Research and Education (SARE) program, with funding from the National Institute of Food and Agriculture, U.S.

Muhammad, I. R., Liyakat, H. M., Tanvir, S., Talal, A., Iqbal, M. I. and Ismail, M. O. (2016). Bacteria and fungi can contribute to nutrients bioavailability and aggregate formation in degraded soils. Microbial Research, 183: 36-41.

Naheeda, B., Cheng, Q., Muhammad, A. A., Sajjad,., Muhammad, I. K. Muhammad, A., Nadeem, A. and Zhang. L. (2019). Role of arbuscular 
mycorrhizal fungi in plant growth regulation: implications in abiotic stress tolerance. Front. Plant Sci., 10(1068): $1-15$.

Nagamani, A., Kunwar, I. K. and Manoharachary, C. (2006). Handbook of soil fungi. I. K. International Pvt. Ltd., New Delhi.

Richard, J., Manuela, P., Antonella, S., Anna, K. and Stanislav, K. (2017). The role of soil microorganisms in plant mineral nutrition - current knowledge and future directions, Front. Plant Sci., 8:1617.

Shitole, S., Kadam, V., Bankar, P., Chandankar, S., Wagh, S., and Kanade, M. B. (2019). Isolation and identification of soil fungi of banana fields from Baramati area of Pune dist of Maharashtra, India. Int. J. Curr. Microbiol. App. Sci., 8(7): 2193-2197.

Sun, J. M., Irzykowski, W., Jedryczka, M. and Han, F. X. (2005). Analysis of the genetic structure of Sclerotina sclerotiorum (lib) de Bary Populations from different regions and host by Random Amplified Polymorphic DNA markers. J. Integr. Plant Biol., 47(4): 385-395.

Treseder, K. K., and Lennon, J. T. (2015). Fungal traits that drive ecosystem dynamics on land. Microbiol. Mol. Biol. Rev, 79: 243-262.

Wagg, C., Bender, S. F., Widmer, F. \& van der Heijden, M. G. A. (2014). Soil biodiversity and soil community composition determine ecosystem multifunctionality. Proceedings of the National Academy of Sciences, 111(14): 5266-5270.

Zifcakova, L., Vetrovsky, T., Howe, A., and Baldrian, P. (2016). Microbial activity in forest soil reflects the changes in ecosystem properties between summer and winter. Environ. Microbiol., 18 (1): 288-301.

\section{How to cite this article:}

Rohit Taware, Pooja Kadam, Anuja Shende, Anuradha Bhosale, M. B. Kanade and Chavan, S. J. 2020. Relationship between Macro and Micronutrients Profile with Fungal Flora of Rhizosphere Soils from Wheat, Maize and Sorghum Fields of Baramati Area. Int.J.Curr.Microbiol.App.Sci. 9(12): 1325-1333. doi: https://doi.org/10.20546/ijcmas.2020.912.163 